\title{
EFECTOS DE LA MARIHUANA EN LA COGNICIÓN: UNA REVISIÓN DESDE LA PERSPECTIVA NEUROBIOLÓGICA
}

\author{
Gladys Torres ${ }^{1,2, a}$, Fabián Fiestas $3, b$
}

\begin{abstract}
RESUMEN
La marihuana es una sustancia psicoactiva ampliamente usada en la sociedad, especialmente entre los más jóvenes. El uso de esta sustancia ha sido asociado consistentemente con diversos problemas de salud, muchos de los cuales tienen en común una alteración en las manifestaciones cognitivas de la conducta, incluyendo la memoria, la atención, la emoción y la toma de decisiones. Se encontró evidencia que los cannabinoides, la sustancia activa de la marihuana, impactan negativamente en la memoria a corto plazo, memoria de trabajo y la toma de decisiones. Asimismo, los cannabinoides afectan la atención y la interacción entre los eventos cognitivos y la emoción. Esta información puede ser usada como argumento de plausibilidad biológica para interpretar una serie de hallazgos clínicos y epidemiológicos en los que el uso de marihuana se ha mostrado relacionado con problemas tales como accidentes de tránsito, psicosis, depresión, pobre trayectoria académica, entre otros.
\end{abstract}

Palabras clave: Abuso de marihuana; Cognición; Conducta; Memoria; Neurobiología (fuente: DeCS BIREME).

\section{EFFECTS OF MARIJUANA ON COGNITION: A REVIEW FORM THE NEUROBIOLOGICAL PERSPECTIVE}

\begin{abstract}
Marijuana is one of the most commonly used psychoactive substances in society, mainly among youths. Its use has been consistently associated with several health problems, many of which have in common an impairment in the cognitive processes of behavior, including the memory, attention, emotion and decision making. There is evidence suggesting that cannabinoids, marijuana's primary psychoactive substance, have a negative effect in short-term memory, working memory, and decision making. It has also been found that cannabinoids affect attention and the interaction between cognitive events and emotion. This information can be used as an argument of biological plausibility to assess clinical and epidemiological research findings that show that marijuana's use is associated to problems such as traffic accidents, psychosis, depression and poor academic records, among others.
\end{abstract}

Key words: Marijuana abuse; Cognition; Behavior; Memory; Neurobiology (source: MeSH NLM).

\section{INTRODUCCIÓN}

La marihuana (sustancia sustraída de la planta Cannabis) es la sustancia psicoactiva ilegal de mayor uso en el mundo. La Organización de las Naciones Unidas (UNODC) ha estimado que en el 2009, entre 125 y 203 millones de personas en el mundo usaron esta sustancia, siendo la prevalencia anual de uso de la droga de 2,8-4,5\% de la población mundial entre 15 y 64 años ${ }^{(1)}$. En el Perú, la prevalencia anual de uso de marihuana ha sido informada en alrededor de $0,7 \%$, la cual es una prevalencia bastante baja comparada con la de otros países vecinos, como Colombia, Bolivia y Chile, donde la prevalencia anual de uso de marihuana es 2,3, 4,3 y $6,7 \%$, respectivamente. Esta discrepancia entre países con similares características socioeconómicas de una misma región puede deberse a diferencias metodológicas, que en este caso pueden estar significando para el Perú un subregistro de consumo de la marihuana (especialmente, cuando la UNODC, publicó una prevalencia anual para el 2005 de 3,3\%). Lo cierto es que de las tendencias globales se sabe que en general el consumo de marihuana incrementa conforme crece el desarrollo de los países ${ }^{(1,2)}$. Si son varios los países en Latinoamérica que van creciendo en su economía sostenidamente, incluyendo al Perú, entonces es de esperar que la tendencia de consumo de marihuana también vaya creciendo en los próximos años.

Son muchos los problemas de salud para los que se ha sugerido que el uso de marihuana podría tener un papel contribuyente, incluyendo problemas pulmonares,

\footnotetext{
Colegio San Ignacio de Recalde, Organización San Ignacio de Loyola. Lima, Perú.

2 Facultad de Medicina, Universidad Nacional Mayor de San Marcos. Lima, Perú.

3 Laboratorios de Investigación y Desarrollo, Universidad Peruana Cayetano Heredia. Lima, Perú.

4 Unidad de Análisis y Generación de Evidencias en Salud Pública, Instituto Nacional de Salud. Lima, Perú.

Bióloga; ${ }^{\mathrm{b}}$ médico epidemiólogo
}

Recibido: 24-01-12 Aprobado: 08-02-12 
cardiovasculares, reproductivos, teratogénicos, y oncológicos, aunque sin evidencia concluyente. Otros problemas de salud con mayor evidencia de asociación, posiblemente causal, incluyen problemas de tipo neurológico, como el deterioro agudo de habilidades de manipulación neuromotora que son necesarias como por ejemplo para conducir vehículos (como procesamiento de información, reacción temporal, coordinación perceptual-motora, acción motora, memoria de trabajo y atención) ${ }^{(3)}$. La literatura epidemiológica es consistente con ello al mostrar asociación entre accidentes de tránsito con el uso de marihuana (4-8), aunque son necesarios más estudios para identificar con precisión efectos independientes de esta sustancia respecto a otras sustancias, principalmente alcohol, ya que el uso concomitante es muy frecuente ${ }^{(9)}$.

Además del abuso y la dependencia, para los que la marihuana es causa necesaria, se ha sugerido que hay otros problemas de salud mental que también podrían estar siendo causados por esta sustancia, e incluyen principalmente la psicosis no-afectiva (especialmente, la esquizofrenia) ${ }^{(10-12)}$ y la depresión ${ }^{(10,13)}$. Entre los problemas psicosociales asociados a la marihuana y que más han interesado a los investigadores está el fracaso en lograr metas académicas. Existe evidencia que muestra asociación entre el consumo de marihuana con un bajo logro académico, especialmente con fracaso para culminar la escuela secundaria a su debido tiempo y con menor nivel de grado académico alcanzado a la edad adulta ${ }^{(14-19)}$.

Entonces, el consumo de marihuana puede estar teniendo serias consecuencias en la sociedad, aunque aún poco conocidas. Una parte considerable de los problemas a la salud y psicosociales que el uso de marihuana podría estar causando, como la mayor probabilidad de sufrir accidentes de tránsito, psicosis, depresión, y problemas educativos, involucran de alguna manera el constructo neuropsicológico conocido como cognición. La cognición es entendida como la conducta intermedia entre la sensación (información que recibimos a través de nuestro sistema sensorial) y la acción (que es nuestra respuesta final a dichas sensaciones). Así, la cognición involucra la interacción de diversas manifestaciones conductuales como la memoria, atención, emoción, lenguaje, pensamiento y conciencia ${ }^{(20,21)}$. Una disrupción en cualquier área podría traducirse en alguno de los problemas de salud y psicosociales que hemos descrito y que han sido relacionados con el uso de marihuana.

El objetivo de esta revisión es hacer una recopilación de los hallazgos más sólidos respecto al efecto que tiene la marihuana en las diferentes manifestaciones de la cognición, centrándonos en la memoria, atención, emoción y toma de decisiones, ya que son los funciones neuropsicológicas más directamente relacionadas con los problemas de salud asociados a la marihuana, descritos previamente. Para ello, se realizó una búsqueda de revisiones y artículos originales de los últimos 20 años usando el buscador de bibliografía biomédica ISIWeb of Science, y se realizó un ordenamiento de las referencias por número de citaciones, escogiéndose para esta revisión aquellas con demostrado nivel de impacto (definido por un número de citaciones mayor a 20 desde que salió la publicación en las revistas indizadas). Se dividió los hallazgos en cuatro secciones que reflejan los criterios de búsqueda usados, los cuales significaron la intersección de marihuana con memoria, atención, emoción y toma de decisiones.

\section{MARIHUANA Y MEMORIA}

Antes de exponer acerca del impacto de la marihuana en la memoria, podría ir en beneficio del lector no especializado en neurociencias que describamos aspectos de la memoria que son muy importantes para su estudio. La memoria tiene tres aspectos que la hacen posible: (i) La codificación o registro, que es el proceso por el cual el cerebro capta un evento a través de las vías sensoriales; (ii) La consolidación, que significa la creación de nuevos neurocircuitos a partir del evento previamente captado; y (iii) la capacidad de recuperar la información a través de eventos neuroquímicos que se suceden en dichos neurocircuitos, lo que se traduce en la experiencia de "recordar" el evento fijado ${ }^{(22)}$. La memoria es cimiento de los procesos cognitivos, y supone una eficiencia en la plasticidad neuronal con respecto a la actividad eléctrica, de neurotransmisores, de segundos mensajeros, y en algunas modificaciones de proteínas sinápticas, para un engranaje saludable de los tres aspectos antes mencionados.

Las diferentes clasificaciones de la memoria derivan de aspectos como su duración, el tipo de información almacenada o en qué estructuras cerebrales están involucradas. En esta revisión, nos centraremos en la duración de la memoria, que es la más típicamente usada en su estudio. Así, la memoria puede ser de largo o de corto plazo.

La memoria a largo plazo (MLP) se distingue por su capacidad de almacenar una gran cantidad de información, indefinidamente. Se divide a su vez en MLP declarativa y en MLP no declarativa. La MLP explícita o declarativa recupera eventos o experiencias del pasado, un hecho histórico o un acontecimiento familiar, y puede ser medida por el pedido del recuerdo o la identificación del mismo; 
puede a su vez, diferenciarse en la memoria semántica (significado de las palabras, hechos, normas), y la memoria episódica (fechas de eventos, lugar y algunas emociones asociadas). La MLP implícita o no declarativa permite el desempeño incluso en ausencia de lo consciente, lo que coloquialmente se reconoce como "lo hice por inercia", por ejemplo como cuando manejamos bicicleta ${ }^{(22)}$.

Por su parte, la memoria a corto plazo (MCP), dura aproximadamente menos de un minuto, tiene un límite de capacidad y corresponde a unas cinco o seis palabras, o siete dígitos. Puede ser borrada por traumas encefálicos o electroconvulsiones, los cuales no afectarían la memoria a largo plazo. La memoria de trabajo es un tipo especial de MCP, ya que también tiene una capacidad limitada para almacenar y manipular información en poco tiempo, pero se diferencia porque manipula la información que guarda. Esto quiere decir, que la memoria de trabajo no solo tiene la imagen de la información a manera de representación mental en su estado actual, sino que también hace la representación de una posible situación futura para poder trabajarla ${ }^{(22)}$.

Estudios en animales y con humanos voluntarios muestran que los cannabinoides, que son la sustancia activa de la marihuana, tienen principalmente efectos observables tanto en la MCP como en la memoria de trabajo ${ }^{(23-26)}$. Sin embargo, la evidencia es aún controversial ya que los estudios que evalúan los efectos de los cannabinoides en la memoria son de alta complejidad y un consenso aún no ha podido ser alcanzado. Es difícil reconocer en los estudios cuáles son efectos de la sustancia y cuáles son por otros factores que los investigadores no pueden controlar o identificar. Así, por ejemplo, en estos estudios debe asegurarse que no haya ninguna afección en el individuo que esté perjudicando su capacidad para recordar información y que haya buen desempeño en los tres eventos de la memoria (registro, consolidación y recuerdo) antes de la administración de la droga.

Otra variable interviniente en los estudios sobre la afección de cannabinoides en la memoria es la cantidad y calidad de la droga a ingerirse y su vía de administración. Los cannabinoides pueden usarse por vía oral, sublingual, por inhalación, intramuscular e intravenosos. Para efectos de esta revisión, nos concentraremos en la administración de inhalación del humo (cigarrillo), por ser el medio más frecuente de uso de la marihuana en nuestro medio. Un cigarro típico puede contener alrededor de $0,35 \mathrm{~g}$ de marihuana, que contiene $0,3-10 \%$ de principio activo delta-9-THC. Al fumar, solo $10-25 \%$ del principio activo entra a circulación, alcanzando un pico máximo de concentración a los 3-10 min. Sin embargo, los efectos psicotrópicos pueden iniciarse a pocos segundos después de fumar, alcanzando su pico máximo de expresión a los 15-30 min y tener una duración de entre 2 a $3 \mathrm{~h}$. Todos estos efectos, dependerán del peso del cigarro, la frecuencia de inhalación, la profundidad del soplo, la extensión de cuánto se mantiene el aliento después de inhalar y hasta de la capacidad vital pulmonar del individuo ${ }^{(22)}$.

Se han realizado algunos estudios del efecto de los cannabinoides en la memoria, que toman en cuenta aspectos metodológicos, la dosis administrada y la existencia de tolerancia, que es una de las características más importantes para definir dependencia a la droga ${ }^{(27,28)}$. Por ejemplo, en un estudio caso-control con sujetos voluntarios consumidores de marihuana y no consumidores realizado para medir el impacto del marihuana en las habilidades básicas de la MCP, se encontró una asociación consistente entre ser usuario de la sustancia y una disrupción en las capacidades de las diferentes vías de la memoria ${ }^{(27)}$.

Block et al.,(27) han descrito además, que para consumidores crónicos frecuentes (siete o más veces a la semana) se observan deficiencias en pruebas de habilidades matemáticas y de expresión verbal. $Y$ utilizando pruebas más específicas (test de Buschke, que mide aprendizaje y recuerdo), se encuentran fallas significativas en la vía de recuerdo de la memoria. Sin embargo, en consumidores menos frecuentes no se encontró asociación entre el consumo de la sustancia y deficiencias en memoria, y los sujetos mostraron únicamente confusión acerca de algunos conceptos. Estos resultados muestran con claridad un efecto de los cannabinoides a dosis altas en las habilidades específicas para recuerdo, pero la evidencia no es clara cuando se trata de dosis bajas. Sin embargo, aún no es completamente claro si estos son efectos por dosis acumulada de vida, efectos de consumo reciente o características prexistentes del individuo.

Por su parte, la evidencia que los cannabinoides afecta negativamente la memoria de trabajo es relativamente sólida ${ }^{(27,29-33)}$, lo cual tiene especial relevancia dado que la memoria de trabajo está alterada en la esquizofrenia ${ }^{(34)}$, siendo así un nexo más con dicha enfermedad. Además, los hallazgos sugieren que los efectos de los cannabinoides en el adulto son mayores por haber una acumulación de dosis a largo plazo y que hay diferencias para el hombre y la mujer en cuanto a los efectos de los cannabinoides en procesos de memoria de trabajo ${ }^{(26,35)}$. Estos hallazgos pueden estar añadiendo evidencia útil para el estudio de la asociación marihuana-esquizofrenia.

En el ámbito molecular, el efecto de los cannabinoides tanto en la memoria de corto plazo, como en 
memoria de trabajo, se puede explicar por afectar específicamente el receptor CB1, el cual tiene un papel importante en la memoria y en otros aspectos de la cognición, así como también en la percepción del dolor ${ }^{(22,36)}$. El receptor CB1 que liga cannabinoides endógenos (endocannabinoides), es un receptor ligado a proteína-G, distribuido principalmente en la corteza prefrontal, con alta densidad en los ganglios basales y el hipocampo ${ }^{(22)}$. Participan además en la regulación de ganglios basales que viene desde el cerebelo, donde los endocannabinoides afectarían las sinapsis glutamatérigcas suprimiendo su excitación al momento de regresar a la neurona presináptica (36). Además, es función de los endocannabinoides actuar como mensajeros retrógrados para neuronas presinápticas, modulando la liberación de los neurotransmisores GABA y glutamato, que por tener efectos opuestos, regularán a su vez la activación de proyecciones neurales postsinápticas. Los endocannabinoides logran inhibir la liberación de neurotransmisores presinápticos actuando sobre los canales de calcio por activación del receptor CB1, y puede dar dos formas diferentes de plasticidad a corto plazo si es la transmisión de GABA o la de glutamato la que esté involucrada respectivamente ${ }^{(37)}$.

GABA es inhibidor por excelencia y se encarga de regular diferentes procesos, como el sueño, modulación de dolor, ansiedad, entre otros. Antes de explicar el rol de los endocannabinoides en la vía del GABA, debemos explicar el concepto de "supresión de la inhibición inducida por depolarización" (SIID). La SIID da cuenta de la supresión de la liberación de GABA por la neurona presináptica luego que la neurona postsináptica, previamente activada, enviara un mensaje de supresión de liberación de GABA a dicha neurona presináptica que generó al inicio la activación de la postsináptica. Esta supresión de la inhibición da como resultado que la neurona postsináptica continúe haciendo plasticidad. Este efecto parece predominar en el hipocampo y cerebelo ${ }^{(37-39)}$. Los endocannabinoides al parecer son los mensajeros que activan esta vía, resultando entonces en una potenciación y generación poco ordenada a largo plazo de la plasticidad en las neuronas postsinápticas ${ }^{(36)}$.

Por otro lado, y antes de explicar el papel de los endocannabinoides en la vía del glutamato, que es activador por excelencia del sistema nervioso, debemos explicar el concepto de "supresión de la excitación inducida por despolarización” (SEID). En este caso, el mensajero enviado por la neurona postsináptica inhibe la liberación de glutamato de la presináptica, que estaba destinada a hacer que la plasticidad de la postsináptica continúe dándose. Este fenómeno, que sucede predominantemente en el cerebelo, tiene como efecto neto un control de la plasticidad de la neurona postsináptica (efecto que es contrario al producido por el SIID). Nuevamente, el mensajero retrógrado que hace posible el SEID, como en el caso del SIID, es un endocannabinoide ${ }^{(37)}$. Entonces, ambas formas de control en la plasticidad sináptica son inducidas por los endocannabinoides, por lo que estos tendrían la función de regular y coordinar las redes neuronales involucradas en procesos fisiológicos como memoria o coordinación motora ${ }^{(37,40,41)}$.

El hipocampo cumple un papel muy importante en la formación de memoria, y el hecho que los endocannabinoides generen una proliferación poco controlada de la plasticidad neuronal a través del SIID, puede explicar en parte los hallazgos que el uso de la marihuana está asociado a una afección de la MCP. Por ejemplo, algunos estudios diseñados para observar la eficiencia de aprendizaje bajo los efectos la marihuana han mostrado que individuos bajo efectos de la sustancia tienen problemas para recordar lo que aprendieron minutos antes del consumo; es decir, tienen problemas de fijar la información en la memoria de corto plazo hasta consolidarlo (dependiendo del tiempo transcurrido entre el consumo y el momento en que recibió la información). Si los cannabinoides promueven la plasticidad neuronal, el cómo es que los individuos tendrían problemas para fijar memoria a corto plazo, estaría asociado a que estas nuevas conexiones se han formado por plasticidad alterada, que no necesariamente son congruentes en las rutas del nuevo aprendizaje ${ }^{(22,27)}$. Al permitir la marihuana una plasticidad promiscua, habrá deficiencias en cognición y recuerdo. En experimentos en ratones knock-out para el receptor CB1 se han observado tanto fallas en la MCP como en la potenciación a largo plazo, probablemente por la inestabilidad de las conexiones neurales formadas ${ }^{(36)}$.

Existen otros dos receptores para cannabinoides: CB2 y CB3. El primero, CB2, está distribuido principalmente en el sistema nervioso periférico y órganos linfoides, y solo se encuentra en pocas cantidades a nivel glial, por lo que no es relevante para fines de estudios cognitivos ${ }^{(22-37)}$. CB3 sí aparece en sistema nervioso central, y sus ligandos inhiben tanto la liberación de glutamato como la potenciación a largo plazo. Al no liberarse glutamato, la neurona postsináptica no logra despolarizarse suficientemente para desbloquear el $\mathrm{Mg}^{2+}$ de los receptores NMDA (N-metil-Daspartato), afectándose así la plasticidad neuronal y, por ende la memoria. Sin embargo, delta-9-THC parece que no es buen ligando para CB3, por lo que los efectos de la marihuana en la memoria parecen ser debidos solo a los receptores $\mathrm{CB} 1{ }^{(36)}$.

En memoria de trabajo, la literatura describe ciertos efectos de los cannabinoides por alterar directamente el complejo del hipocampo reduciendo su habilidad de codificación, dando como resultado: torpeza e 
inexactitud ${ }^{(42,43)}$. De manera interesante, debido al potencial impacto en el aprendizaje de las personas en edad escolar, Pattij (26), ha observado que cuando se usa el rimonabant (molécula que comercialmente se usa para inhibir el apetito y que al ser antagonista de CB1 permite medir su desempeño), las fallas en memoria de trabajo medida por pruebas de reconocimiento tienen resultados más agudos en adolescentes que en adultos. Finalmente, para una mejor comprensión de la cognición reproducida por memoria de trabajo, se debe tomar en cuenta que esta está regulada por la emoción y la atención ${ }^{(44,45)}$, procesos que se describirán más adelante.

\section{MARIHUANA Y ATENCIÓN}

El cerebro humano, en su origen evolutivo animal, busca comportarse con una reglamentación que Miller (28) llama "de abajo hacia arriba", en el que el proceso va de los mecanismos moleculares (dadas por interacciones físicas y químicas), a los niveles de comunicación celular, hasta llegar al control del comportamiento humano. De esta manera, nuestras funciones homeostáticas que ajustan nuestras funciones biológicas a las necesidades ambientales (que algunos llaman "el instinto"), rigen nuestro comportamiento y desarrollo. Sin embargo, como seres sociales, la mayoría de nuestras conductas están reguladas por la naturaleza de los estímulos sensoriales que se conectan con las respectivas rutas neuronales de respuesta, es decir "arriba hacia abajo". Siendo nuestro medio externo y la interacción con nuestros semejantes lo que nos permite tener control cognitivo para seleccionar respuestas de acuerdo a nuestros intereses (atención selectiva) y mediar nuestro comportamiento ${ }^{(28)}$.

Cuando hay un conflicto entre diferentes vías con diferentes fuentes de información, se crea una competencia para la expresión del comportamiento y ganará aquel estímulo con la fuente de apoyo más fuerte. Esto es claramente expresado usando medidas neuropsicológicas, como la técnica de Stroop, en la que se crean conflictos en la atención al escribir el nombre de un color en letras de otro color y se le pide a un sujeto que diga el color de lo que está escrito. La vía más fuerte es la parte cognitiva ya desarrollada, que lleva al sujeto a leer la palabra en lugar de dejarse guiar por el sentido visual solamente y mencionar el color que está viendo, mas no el que lee. Si el individuo ha puesto atención en las indicaciones, por más ligero que sea el conflicto, logrará responder lo que se le ha solicitado dando uso a la vía sensorial por la encima de la cognitiva ${ }^{(28)}$. La competencia entre estas vías cognitivas y sensoriales, son el resultado evolutivo único en humanos, que responde a situaciones específicas, como presión social, percepción de los objetos externos, acción del cuerpo, incluyendo las interacciones sociales ${ }^{(46)}$.

Respecto al efecto de la marihuana en la atención, los estudios muestran que ésta causa una alteración de la atención transitoria que es evidente a los siete días de haber consumido, pero que desaparece a los 28 días ${ }^{(47,48)}$. Además, estudios con usuarios de cannabis comparados con no-usuarios donde no se han encontrado diferencias en las pruebas neuropsicológicas con una de atención atribuible a un uso crónico ${ }^{(49)}$. Estos últimos hallazgos han sido consistentes con ulteriores hallazgos neurobiológicos. Por ejemplo, Jager ${ }^{(24)}$ realizó estudios para ver el efecto de la marihuana en tareas de memoria asociativa y atención, usando una técnica de morfometría, en la cual se identifican las diferencias en la composición del tejido según la región por densidad de tejido vía resonancia magnética funcional, en que podría demostrarse si existe algún tipo de cambio morfométrico en la tridimensionalidad de las neuronas. El estudio comparó el desempeño durante la realización de tareas de atención y memoria asociativa, al solicitar a los individuos (consumidor y no-consumidor) que conecten dos figuras. No se encontraron diferencias significativas, lo cual sería consistente con la hipótesis de que la marihuana no altera la atención ${ }^{24)}$. Sin embargo, si bien aún no está claro que el consumo de marihuana afecte la atención específicamente, al estar relacionada con otros procesos neurocognitivos, la atención podría verse afectada como un efecto secundario de fallas en la memoria o regulación de la emoción como se revisará a continuación.

\section{MARIHUANA Y EMOCIÓN}

Todos los eventos y objetos que forman parte de nuestra vida diaria suceden en representaciones neurales de diversas modalidades. Las interacciones entre la percepción, la acción del cuerpo, el ambiente, el estado del ánimo y otros agentes serán de gran importancia al momento de realizar una acción ${ }^{(46)}$.

Actualmente, se conoce que las emociones influyen en la atención y percepción según los estímulos sociales y la retroalimentación de respuestas. Phelps ${ }^{(44)}$, describe el objetivo de la psicología cognitiva como "la forma como el hombre colecta, almacena, modifica e interpreta la información; o la información ya almacenada internamente". A este concepto debe sumársele el papel de la emoción, cuyo neurosustrato es el sistema límbico: la amígdala, principalmente. Se ha descrito que los circuitos neuronales de la emoción y la cognición interactúan constantemente, desde los sistemas de percepción más primitivos hasta los de la toma de decisiones y el razonamiento ${ }^{(44)}$. En la cognición, la emoción es un componente muy importante 
que interviene en la formación y recolección de memoria episódica, alterando los tres componentes de la memoria (registro, codificación y recuerdo) ${ }^{(28)}$.

La amígdala regula el registro del estímulo a recordarse, limitando la atención solo a los detalles internos que cada persona considera de mayor importancia para ella misma (de acuerdo a sus propias necesidades, vacíos o satisfacciones que se tiene como personalidad individual). Entonces, la función de la amígdala no sería el registro en sí, sino la modulación (o modificación) del neurocircuito que permite el recuerdo. Una muestra clara y muy típica de esto son los eventos que resultan de una respuesta emocional y que siendo muy importantes para la supervivencia, son más difíciles de olvidar ${ }^{(44)}$. Por ejemplo, aquellos que sobrevivieron al pasado terremoto de Pisco (Ica-Perú) en el 2007, van a recordar nítidamente dónde y qué estuvieron haciendo en el momento del sismo. Tanto está esto demostrado, que la memoria episódica no puede medirse en la precisión del recuerdo, ya que hay un alto grado de emotividad en el circuito de la recuperación de la memoria que ésta puede no reflejar con precisión real y exacta lo sucedido, ya que además integrará al recuerdo lo subjetivamente vivido, lo que puede añadir adornos y fantasías en el recuerdo ${ }^{(28,44,46)}$.

Entre las varias funciones de los cannabinoides endógenos está el control homeostático de las emociones y la regulación del comportamiento motivado el cual, como hemos descrito anteriormente, se guía por la atención ${ }^{(37)}$. El sistema natural de cannabinoides está distribuido en el complejo amigdalar y se ha comprobado su control sobre las emociones con estudios del comportamiento después de la administración de antagonistas de los receptores cannabinoides y los déficits de recompensa observados en ratones knock-out para CB1 ${ }^{(37)}$. Sanchis et al., ${ }^{(50)}$ han descrito que el control que ejercen los receptores CB1 no solo estaría dado a los sistemas de recompensa como comportamiento motivado, sino que los receptores CB1 estarían también involucrados en el control de la recompensa homeostática, satisfacción de las necesidades básicas (comer, dormir, por ejemplo). Por lo que los receptores para cannabinoides estarían asociados no solo a disturbios en la motivación, sino también al proceso emocional de la información percibida del medio ${ }^{(50)}$. Este hallazgo es especialmente interesante puesto que se ha postulado que uno de los mecanismos por los que la marihuana está asociada a una trayectoria educativa pobre es a través del así llamado síndrome amotivacional, donde el individuo pierde interés en las cosas de la vida cotidiana como efecto del consumo crónico de la sustancia ${ }^{(18,51)}$.

La manera en que los endocannabinoides pueden causar desmotivación es a través de una disminución de la liberación de glutamato y de factores de liberación de corticotropinas, reduciendo las vías de entrada al complejo amigdalar. El límite entre la exposición a una dosis significativa de cannabinoides para el equilibrio final del este sistema, llevaría a la ansiedad o ansiolisis, dependiendo de la tasa de activación de la amígdala con proyecciones al hipocampo y al tronco encefálico, siendo la ansiolisis la respuesta más probable a un incremento de la transmisión cannabinoide en este sistema (37).

\section{MARIHUANA Y TOMA DE DECISIONES}

La toma de decisiones se define como un proceso mental, de orden cognitivo alto, en el que se debe seleccionar una acción apropiada de entre diferentes escenarios alternativos e inhibir los comportamientos inapropiados ${ }^{(26)}$. Este proceso cognitivo es de gran importancia en la cognición, ya que las fallas en este sistema podrían conllevar a comportamientos impulsivos o desórdenes de atención e hiperactividad.

Para medir estos procesos, la mayoría de estudios usan los sistemas de recompensa inmediata e impulsividad, midiendo en las pruebas la incapacidad de inhibir el comportamiento a manera que se incrementa la preferencia por la recompensa. Se ha descrito que el uso crónico de marihuana incrementa la probabilidad de optar por los comportamientos asociados a mayor riesgo. Por ejemplo, al escoger entre dos opciones para aumentar las ganancias monetarias, los sujetos que usan marihuana tienden a escoger las opciones asociadas a grandes pérdidas de dinero, comportamiento que sugiere que estos sujetos toman decisiones riesgosas impulsivamente ${ }^{(52-54)}$.

Churchwell et al. proporcionan una explicación anátomomorfológica para esta conducta impulsiva, con los descubrimientos de un sofisticado estudio basado en los hallazgos de Fuster et al. y Ellgren et al. Los primeros, Fuster et al., encontraron que la morfogénesis en la corteza prefrontal potencia la capacidad de organizar temporalmente los planes de acción y elecciones al momento de lograr objetivos ${ }^{(55)}$. Por su parte, Ellgren et al., encontraron en modelos animales que la densidad del receptor CB1 en la corteza prefrontal cambia durante la adolescencia, sugiriendo que esta etapa representa un período de vulnerabilidad morfológica a la marihuana ${ }^{(56)}$. Con esta base, Churchwell et al. (57), encontraron que el volumen de la corteza prefrontal medio-orbital de sujetos que abusan del consumo de marihuana es menor comparado con controles sin abuso de esta sustancia. Este hallazgo confluyó además, con una menor capacidad en la función neuropsicológica de orientación futura en los sujetos que abusan de la marihuana, de quien no lo hacen. Todo ello es fuerte evidencia 
conductual que cuenta con sustrato neurobiológico de que aquellos que abusan de marihuana tienen una pobre capacidad de controlar impulsos. Más aun, si el abuso sucede en la adolescencia, cuando la corteza prefrontal aún está en proceso de maduración, entonces esta capacidad puede ser más afectada ${ }^{(57)}$

Sin embargo, los estudios aún no revelan un efecto claro sobre la toma de decisiones en personas consumidoras casuales, consumidores frecuentes de dosis bajas, o consumidores de dosis altas en el largo plazo. Kelleher et al., describen resultados que indican que los consumidores de marihuana de largo plazo presentan un déficit en el desarrollo de tareas de procesamiento de información (tienen lentitud para decidir), pero que esta deficiencia parece normalizarse al pasar los efectos de la intoxicación aguda ${ }^{(25)}$. Se precisa más investigación en esta área, puesto que ello puede estar relacionado luego con una mayor facilidad de los jóvenes que inician marihuana a relacionarse con pares con problemas de conducta o a involucrarse en comportamientos marginales.

\section{CONCLUSIÓN}

La marihuana es una sustancia psicoactiva ampliamente usada en la sociedad, especialmente entre los más jóvenes. El uso de esta sustancia ha sido asociado consistentemente con diversos problemas de salud, muchos de los cuales tienen en común una alteración en las manifestaciones cognitivas de la conducta, incluyendo la memoria, la atención, la emoción y la toma de decisiones. Existe evidencia bien documentada que los cannabinoides, la sustancia activa de la marihuana, impacta negativamente en la memoria a corto plazo, memoria de trabajo y la toma de decisiones. Asimismo, los cannabinoides afectan temporalmente la atención y la interacción entre los eventos cognitivos y la emoción. Estos hallazgos ayudan a interpretar evidencia clínica y epidemiológica de problemas como accidentes de tránsito, psicosis, depresión, pobre trayectoria educativa entre otras dificultades con los que el uso de marihuana se ha encontrado asociado.

\section{Contribuciones de autoría}

FF concibió la idea de investigación y estableció los criterios de búsqueda. GT y FF revisaron la literatura. GT escribió el primer borrador del manuscrito. FF revisó críticamente el manuscrito. GT y FF aprueban la versión final del artículo.

\section{Conflictos de interés}

Los autores declaran no tener conflictos de interés.

\section{REFERENCIAS BIBLIOGRÁFICAS}

1. United Nations Office for Drug Control and Crime Prevention. World drug report 2011. Vienna: UNODC; 2011.
2. Degenhardt L, Chiu WT, Sampson N, Kessler RC, Anthony JC, Angermeyer M, et al. Toward a global view of alcohol, tobacco, cannabis, and cocaine use: findings from the WHO World Mental Health Surveys. PLoS Med. 2008;5(7):e141.

3. Hall W, Degenhardt L. Adverse health effects of non-medical cannabis use. Lancet. 2009;374(9698):1383-91.

4. Gerberich SG, Sidney S, Braun BL, Tekawa IS, Tolan KK, Quesenberry CP. Marijuana use and injury events resulting in hospitalization. Ann Epidemiol. 2003;13(4):230-7.

5. Mura P, Kintz P, Ludes B, Gaulier JM, Marquet $P$, MartinDupont $S$, et al. Comparison of the prevalence of alcohol, cannabis and other drugs between 900 injured drivers and 900 control subjects: results of a French collaborative study. Forensic Sci Int. 2003;133(1-2):79-85.

6. Ramaekers JG, Berghaus G, van Laar M, Drummer $\mathrm{OH}$. Dose related risk of motor vehicle crashes after cannabis use. Drug Alcohol Depend. 2004;73(2):109-19.

7. Laumon B, Gadegbeku B, Martin JL, Biecheler MB. Cannabis intoxication and fatal road crashes in France: population based case-control study. BMJ. 2005;331(7529):1371.

8. Grotenhermen F, Leson G, Berghaus G, Drummer $\mathrm{OH}$, Kruger HP, Longo M, et al. Developing limits for driving under cannabis. Addiction. 2007;102(12):1910-7.

9. Fiestas F, Radovanovic M, Martins SS, Medina-Mora ME, Posada-Villa J, Anthony JC. Cross-national differences in clinically significant cannabis problems: epidemiologic evidence from 'cannabis-only' smokers in the United States, Mexico, and Colombia. BMC Public Health. 2010;10:152.

10. Moore TH, Zammit S, Lingford-Hughes A, Barnes TR, Jones PB, Burke M, et al. Cannabis use and risk of psychotic or affective mental health outcomes: a systematic review. Lancet. 2007;370(9584):319-28.

11. Degenhardt L, Hall W, Lynskey M. Testing hypotheses about the relationship between cannabis use and psychosis. Drug Alcohol Depend. 2003;71(1):37-48.

12. Degenhardt $L$. The link between cannabis use and psychosis: furthering the debate [editorial]. Psychol Med. 2003;33(1):3-6.

13. de Graaf $R$, Radovanovic $M$, van Laar $M$, Fairman $B$, Degenhardt L, Aguilar-Gaxiola S, et al. Early cannabis use and estimated risk of later onset of depression spells: Epidemiologic evidence from the population-based World Health Organization World Mental Health Survey Initiative. Am J Epidemiol. 2010;172(2):149-59.

14. Lee S, Tsang A, Breslau J, Aguilar-Gaxiola S, Angermeyer M, Borges $\mathrm{G}$, et al. Mental disorders and termination of education in high-income and low- and middle-income countries: epidemiological study. Br J Psychiatry. 2009;194(5):411-7.

15. Breslau J, Miller E, Joanie Chung WJ, Schweitzer JB. Childhood and adolescent onset psychiatric disorders, substance use, and failure to graduate high school on time. $J$ Psychiatr Res. 2011;45(3):295-301.

16. Breslau J, Lane M, Sampson N, Kessler RC. Mental disorders and subsequent educational attainment in a US national sample. J Psychiatr Res. 2008;42(9):708-16.

17. Borges G, Mora-Icaza ME, Benjet C, Lee S, Lane M, Breslau $\mathrm{J}$. Influence of mental disorders on school dropout in Mexico. Rev Panam Salud Publica. 2011;30(5):477-83.

18. Fergusson DM, Horwood LJ, Beautrais AL. Cannabis and educational achievement. Addiction. 2003;98(12):1681-92.

19. Fergusson DM, Boden JM. Cannabis use and later life outcomes. Addiction. 2008;103(6):969-76; discussion 77-8. 
20. Mesulam MM. From sensation to cognition. Brain. 1998;121 (Pt 6):1013-52.

21. Fernandez-Serrano MJ, Perez-Garcia M, Verdejo-Garcia A. What are the specific vs. generalized effects of drugs of abuse on neuropsychological performance? Neurosci Biobehav Rev. 2011;35(3):377-406.

22. Ranganathan M, D'Souza DC. The acute effects of cannabinoids on memory in humans: a review. Psychopharmacology (Berl). 2006;188(4):425-44.

23. Fried PA, Watkinson B, Gray R. Neurocognitive consequences of marihuana--a comparison with pre-drug performance. Neurotoxicol Teratol. 2005;27(2):231-9.

24. Jager G, Van Hell HH, De Win MM, Kahn RS, Van Den Brink W, Van Ree JM, et al. Effects of frequent cannabis use on hippocampal activity during an associative memory task. Eur Neuropsychopharmacol. 2007;17(4):289-97.

25. Kelleher LM, Stough C, Sergejew AA, Rolfe T. The effects of cannabis on information-processing speed. Addict Behav. 2004;29(6):1213-9.

26. Pattij T, Wiskerke J, Schoffelmeer AN. Cannabinoid modulation of executive functions. Eur $\mathrm{J}$ Pharmacol. 2008;585(2-3):458-63.

27. Block RI, Ghoneim MM. Effects of chronic marijuana use on human cognition. Psychopharmacology (Berl). 1993;110(12):219-28.

28. Miller EK, Cohen JD. An integrative theory of prefrontal cortex function. Annu Rev Neurosci. 2001;24:167-202.

29. Pope HG, Jr., Yurgelun-Todd D. The residual cognitive effects of heavy marijuana use in college students. JAMA. 1996;275(7):521-7.

30. Fletcher JM, Page JB, Francis DJ, Copeland K, Naus MJ, Davis CM, et al. Cognitive correlates of long-term cannabis use in Costa Rican men. Arch Gen Psychiatry. 1996;53(11):1051-7.

31. Solowij N, Stephens RS, Roffman RA, Babor T, Kadden R, Miller $M$, et al. Cognitive functioning of long-term heavy cannabis users seeking treatment. JAMA. 2002;287(9):1123-31.

32. Bolla KI, Brown K, Eldreth D, Tate K, Cadet JL. Doserelated neurocognitive effects of marijuana use. Neurology. 2002;59(9):1337-43.

33. Kanayama G, Rogowska J, Pope HG, Gruber SA, YurgelunTodd DA. Spatial working memory in heavy cannabis users: a functional magnetic resonance imaging study. Psychopharmacology (Berl). 2004;176(3-4):239-47.

34. Freedman R. Schizophrenia. N Engl J Med. 2003;349(18):1738-49.

35. O'Shea M, Singh ME, McGregor IS, Mallet PE. Chronic cannabinoid exposure produces lasting memory impairment and increased anxiety in adolescent but not adult rats. J Psychopharmacol. 2004;18(4):502-8.

36. Wilson RI, Nicoll RA. Endocannabinoid signaling in the brain. Science. 2002;296(5568):678-82.

37. Rodriguez de Fonseca F, Del Arco I, Bermudez-Silva FJ, Bilbao A, Cippitelli A, Navarro M. The endocannabinoid system: physiology and pharmacology. Alcohol Alcohol. 2005;40(1):2-14.

38. Llano I, Leresche N, Marty A. Calcium entry increases the sensitivity of cerebellar Purkinje cells to applied GABA and decreases inhibitory synaptic currents. Neuron. 1991;6(4):565-74.

39. Pitler TA, Alger BE. Postsynaptic spike firing reduces synaptic GABAA responses in hippocampal pyramidal cells. J Neurosci. 1992;12(10):4122-32.
40. Wilson RI, Kunos G, Nicoll RA. Presynaptic specificity of endocannabinoid signaling in the hippocampus. Neuron. 2001;31(3):453-62.

41. Diana MA, Marty A. Endocannabinoid-mediated short-term synaptic plasticity: depolarization-induced suppression of inhibition (DSI) and depolarization-induced suppression of excitation (DSE). Br J Pharmacol. 2004;142(1):9-19.

42. Egerton A, Allison C, Brett RR, Pratt JA. Cannabinoids and prefrontal cortical function: insights from preclinical studies. Neurosci Biobehav Rev. 2006;30(5):680-95.

43. Riedel G, Davies SN. Cannabinoid function in learning, memory and plasticity. Handb Exp Pharmacol. 2005(168):445-77.

44. Phelps EA. Emotion and cognition: insights from studies of the human amygdala. Annu Rev Psychol. 2006;57:27-53.

45. Ochsner KN, Phelps E. Emerging perspectives on emotioncognition interactions. Trends Cogn Sci. 2007;11(8):317-8.

46. Barsalou LW. Grounded cognition. Annu Rev Psychol. 2008;59:617-45.

47. Pope HG, Jr., Gruber AJ, Yurgelun-Todd D. Residual neuropsychologic effects of cannabis. Curr Psychiatry Rep. 2001;3(6):507-12.

48. Pope HG, Jr., Gruber AJ, Hudson JI, Huestis MA, YurgelunTodd D. Neuropsychological performance in long-term cannabis users. Arch Gen Psychiatry. 2001;58(10):909-15.

49. Gouzoulis-Mayfrank E, Daumann J, Tuchtenhagen F, Pelz S, Becker S, Kunert HJ, et al. Impaired cognitive performance in drug free users of recreational ecstasy (MDMA). J Neurol Neurosurg Psychiatry. 2000;68(6):719-25.

50. Sanchis-Segura C, Cline BH, Marsicano G, Lutz B, Spanagel R. Reduced sensitivity to reward in CB1 knockout mice. Psychopharmacology (Berl). 2004;176(2):223-32.

51. Lynskey M, Hall W. The effects of adolescent cannabis use on educational attainment: a review. Addiction. 2000;95(11):1621-30.

52. Fridberg DJ, Queller S, Ahn WY, Kim W, Bishara AJ, Busemeyer JR, et al. Cognitive Mechanisms Underlying Risky Decision-Making in Chronic Cannabis Users. J Math Psychol. 2010; 54(1):28-38.

53. Lane SD, Cherek DR, Tcheremissine OV, Lieving LM, Pietras CJ. Acute marijuana effects on human risk taking. Neuropsychopharmacology. 2005;30(4):800-9.

54. Lane SD, Yechiam E, Busemeyer JR. Application of a computational decision model to examine acute drug effects on human risk taking. Exp Clin Psychopharmacol. 2006;14(2):254-64.

55. Fuster JM. Frontal lobe and cognitive development. J Neurocytol. 2002;31(3-5):373-85.

56. Ellgren M, Artmann A, Tkalych O, Gupta A, Hansen HS, Hansen $\mathrm{SH}$, et al. Dynamic changes of the endogenous cannabinoid and opioid mesocorticolimbic systems during adolescence: THC effects. Eur Neuropsychopharmacol. 2008;18(11):826-34.

57. Churchwell JC, Lopez-Larson M, Yurgelun-Todd DA. Altered frontal cortical volume and decision making in adolescent cannabis users. Front Psychol. 2010;1:225.

Correspondencia: Fabián Fiestas

Dirección: Av. Honorio Delgado 430. Lima 31, Perú.

Teléfono: (511) 985765743

Correo electrónico: ffiestas@epi.msu.edu 